\title{
Research of Deformation Prediction Method on Large-sized Structure Component Based on Time Series Analysis
}

\author{
GUO Ya-Jing ${ }^{1, a^{*}}$, ZHU Xiao-Rong ${ }^{1, b}$, LU Er-Bao ${ }^{1, c}$,LIU Xu-Liang ${ }^{1, d}$ and HE \\ Cheng-Han ${ }^{1, \mathrm{e}}$
}

\author{
${ }^{1}$ Beijing Research Institute of Precise Mechatronics and Controls, Beijing, 100076, China \\ agyj_buaa@163.com, 'zxrtju00@126.com, 'luerbao@gmail.com, 'dzivencool8@126.com, \\ ehechh2@163.com, "corresponding author
}

\begin{abstract}
Keywords: Large-sized structure, Time series model, Radial-Basis Function (RBF) neural network, Short-term deformation forecasting.
\end{abstract}

\begin{abstract}
Large-sized flexible structure is widely used in industrial production areas, such as aircrafts, automobiles, ships and so on. There will be dynamic deformation in working process which will affect the normal use of structure carrier. In addition, current deformation measurement methods have certain hysteresis, so the research on deformation prediction method must be carried out to forecast deformation trend. Firstly deformation mathematical model of large-sized structure based on mechanism is analyzed. Secondly the deformation trend time series model of large-sized structure is researched. Then the Radial-Basis Function (RBF) neural network is used to complete modeling and training process, to realize short-term deformation forecasting of large-sized structure. The simulation result show that the prediction error is limited within the range of $0.02 '$.
\end{abstract}

\section{Introduction}

Large-sized flexible structure is widely used in industrial production areas, such as aircrafts, automobiles, ships and so on. The detection of dynamic deformation in working process, is particularly important [1][2]. Dynamic deformation will affect the normal use of the structure carrier, therefore it is necessary to detect and predict the dynamic deformation angle, and gasp the trend of deformation in a short time [3]. With the development of real-time deformation measurement technology theory, there is more and more study on inertial device matching measurement technology. The dynamic deformation angle of large-sized flexible structure can be estimated by matching inertial parameters [4][5]. But inertial device matching technology have certain hysteresis, which cannot forecast deformation trend [6].

Because large-sized flexible structure's deformation has the dynamic random characteristics of short changing cycle and uncertain changing trend, the linear model fitting prediction method has lower accuracy and the deformation prediction method has limitation. Radial-Basis Function (RBF) neural network can ignore the specific physical parameters in the process and system, and has the advantages of strong generalization ability [7][8].Therefore RBF neural network is used in this paper to establish large-sized structure's deformation trend time series model, and realize short-term deformation forecasting of large-sized structure.

\section{Deformation Mathematic Model of Large-Sized Structure}

The deformation angle of large-sized structure can be divided into two kinds qualitatively: static deformation angle and dynamic deformation angle. Static deformation angle is not absolute constant, but its changing cycle is usually longer. So generally some structural mechanics methods can be used to model and compensate static deformation angle. While dynamic deformation angle, generated due to large-sized structure's external force and change, has shorter changing cycle. In this paper, just the dynamic deformation prediction method is studied.

Dynamic deformation has the random characteristics of short changing cycle and uncertain changing trend. In the current theory, dynamic deformation is usually considered as two-step Markov 
process energized by white noise. Assume that dynamic deformation angle is $\xi_{i}(\mathrm{t})(i=x, y, z)$, which is independent of other direction. Deformation rate is $\eta(\mathrm{t})=\dot{\xi}(\mathrm{t})$. Then the dynamic deformation model can be expressed as:

$$
\left\{\begin{array}{l}
\dot{\eta}_{x}(\mathrm{t})+2 \beta_{x} \eta_{x}(\mathrm{t})+\beta_{x}^{2} \xi_{x}(\mathrm{t})=\rho_{x}(\mathrm{t}) \\
\dot{\eta}_{y}(\mathrm{t})+2 \beta_{y} \eta_{y}(\mathrm{t})+\beta_{y}^{2} \xi_{y}(\mathrm{t})=\rho_{y}(\mathrm{t}) \\
\dot{\eta}_{z}(\mathrm{t})+2 \beta_{z} \eta_{z}(\mathrm{t})+\beta_{z}^{2} \xi_{z}(\mathrm{t})=\rho_{z}(\mathrm{t})
\end{array}\right.
$$

Where, $\tau_{i}$ is the related time and $\beta_{i}=2.146 / \tau_{i}, \rho_{i}(\mathrm{t})$ is white noise, $\boldsymbol{Q}_{n}=4 \beta_{i}^{2} \sigma_{i}^{2}$ is spectral density, $\sigma_{i}^{2}$ is the variance of deformation angle.

According to the empirical formula, the maximum longitudinal deformation deflection of large-sized flexible structure is related with longitudinal length:

$\boldsymbol{f}_{\max }=\boldsymbol{L} / 500$

Where, $L$ is the longitudinal length, $\boldsymbol{f}_{\max }$ is the maximum longitudinal deformation deflection. Assume that $\boldsymbol{L}=10 \mathrm{~m}$, then $\boldsymbol{f}_{\max }=0.02 \mathrm{~m}$. So the maximum deformation angle of A and B points is:

$$
\varphi_{\max }=0.02 /(10 / 2)=4 \times 10^{-3} \mathrm{rad}
$$

Where, $\varphi_{\max }$ is the maximum deformation angle.

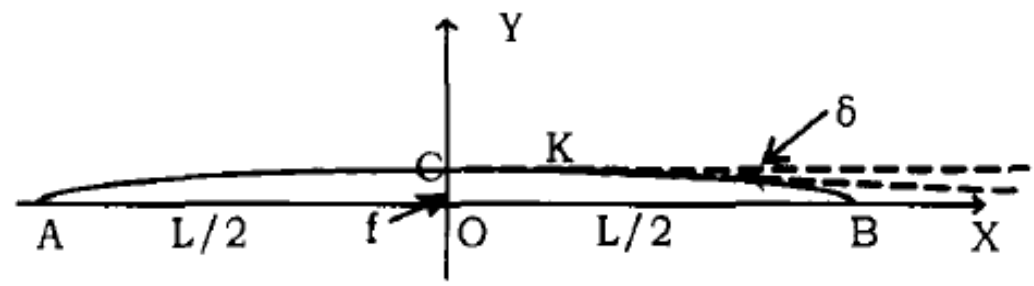

Fig.1 The deformation deflection curve of large-sized flexible structure

Suppose the longitudinal deformation deflection curve is a circular arc, the maximum deformation angle of any point can be calculated by ellipse fitting equation. The ellipse fitting equation of A-C-B arc is:

$$
\frac{x^{2}}{5^{2}}+\frac{y^{2}}{0.02^{2}}=1
$$

Assuming that $\mathrm{K}$ point is located at $1 \mathrm{~m}$, its maximum deformation angle is calculated:

$$
\delta_{\mathrm{K} \max }=1.96 \times 10^{-2} \text { rad }
$$

\section{Deformation Prediction Method Of Large-Sized Structure Based on Time Series Model}

Time Series Model. The deformation mathematical model of large-sized flexible structure is shown in equation (1). The deformation, caused by the comprehend influence of various factors, has a random nonlinear relationship with time affected by the trend of certain factors. So according to the past changing trend, the deformation angle can be forecasted in a short period of time by mathematical statistics method, to realize short-term prediction of dynamic deformation. Therefore the time series model of large-sized flexible structure' deformation is established:

$$
\varphi(\mathrm{t})=\boldsymbol{f}(\varphi(\mathrm{t}-1), \varphi(\mathrm{t}-2), \ldots, \varphi(\mathrm{t}-\mathrm{N}))+\varepsilon
$$

Where, $\mathrm{N}$ is the prediction period, $\varphi(\mathrm{t})$ is the deformation angle to be predicted, $\varepsilon$ is the prediction error, $\boldsymbol{f}(\bullet)$ is the prediction function. 
Time Series Model Based On RBF Neural Network. Large-sized flexible structure's dynamic deformation has the random characteristics of short changing cycle and uncertain changing trend, so linear model fitting prediction method has lower accuracy. Neural network method can ignore the specific physical parameters in the process or system, and establish the complex nonlinear mapping relationship between input and output by studying and training samples, which has the strong generalization ability. RBF neural network is a forward network with great performance. It can realize the best global approximation, its training is easy and quick, and there is no local optimum. The prediction of dynamic deformation can be realized by establishing the functional relationship between deformation angle to be predicted and former N moments' deformation angles.

The time series model based on RBF neural network is shown in Fig.2.

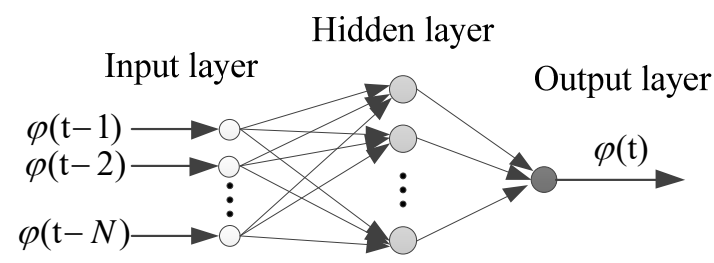

Fig.2 RBF neural network

Where input vector is $\boldsymbol{x}=(\varphi(\mathrm{t}-1), \varphi(\mathrm{t}-2), \ldots, \varphi(\mathrm{t}-\boldsymbol{N}))$, output vector is $\boldsymbol{y}=\varphi(\mathrm{t})$.

\section{Simulation and Verification}

Take the pitching deformation angle for example to simulate and verify the deformation prediction method, because the other two predictions' regularities are the same. K point located at $1 \mathrm{~m}$ is took as an example. The pitching deformation angle of 100 seconds in two-step Markov modelis produced, shown in Fig.3.

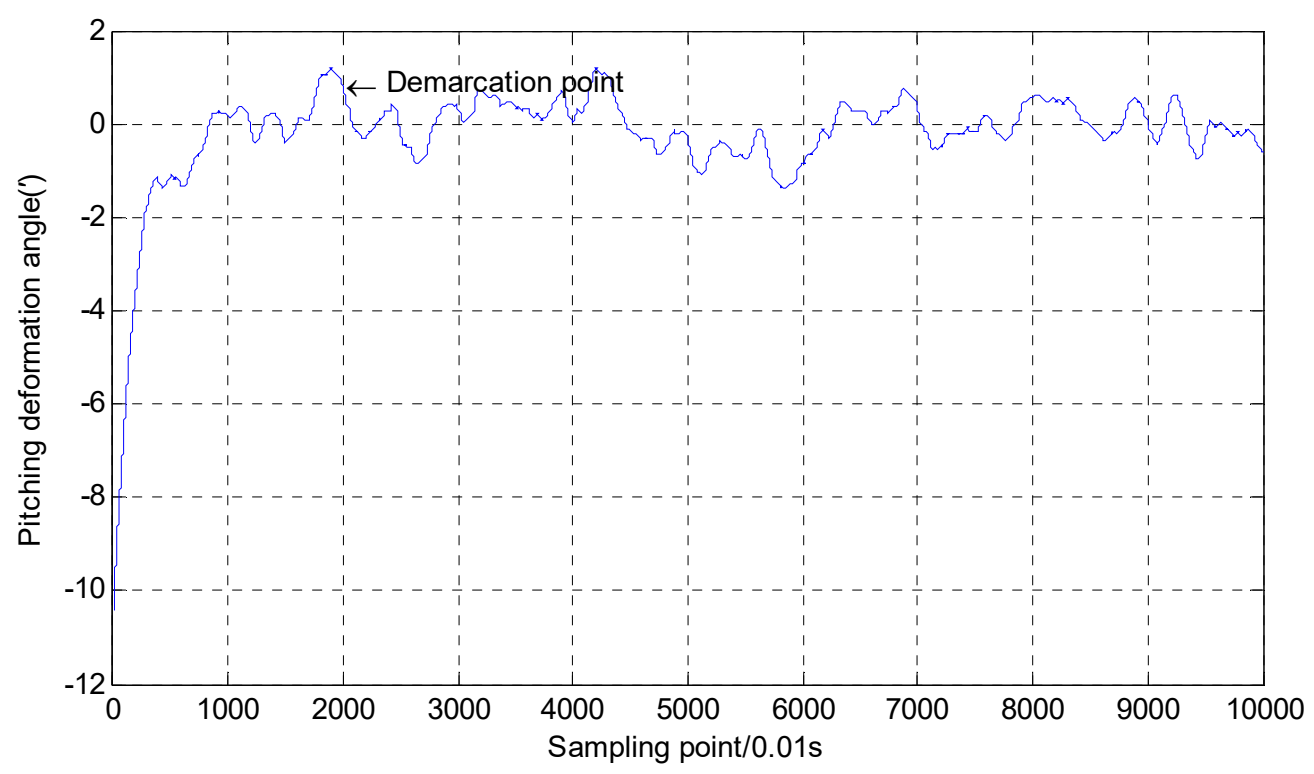

Fig. 3 The pitching deformation angle of 100 seconds

The pitching deformation angle data is used to established the time series model based on RBF neural network, between $\boldsymbol{x}=(\varphi(\mathrm{t}-1), \varphi(\mathrm{t}-2), \varphi(\mathrm{t}-3), \varphi(\mathrm{t}-4))$ and $\boldsymbol{y}=\varphi(\mathrm{t})$. Where the prediction cycle is chosen as $N=4$, RBF neural network fitting error is 0.005 . The former 40 s data is used to train shown in Fig.4. While last 60s data is used to verify, and the test data is shown in Fig.5. 

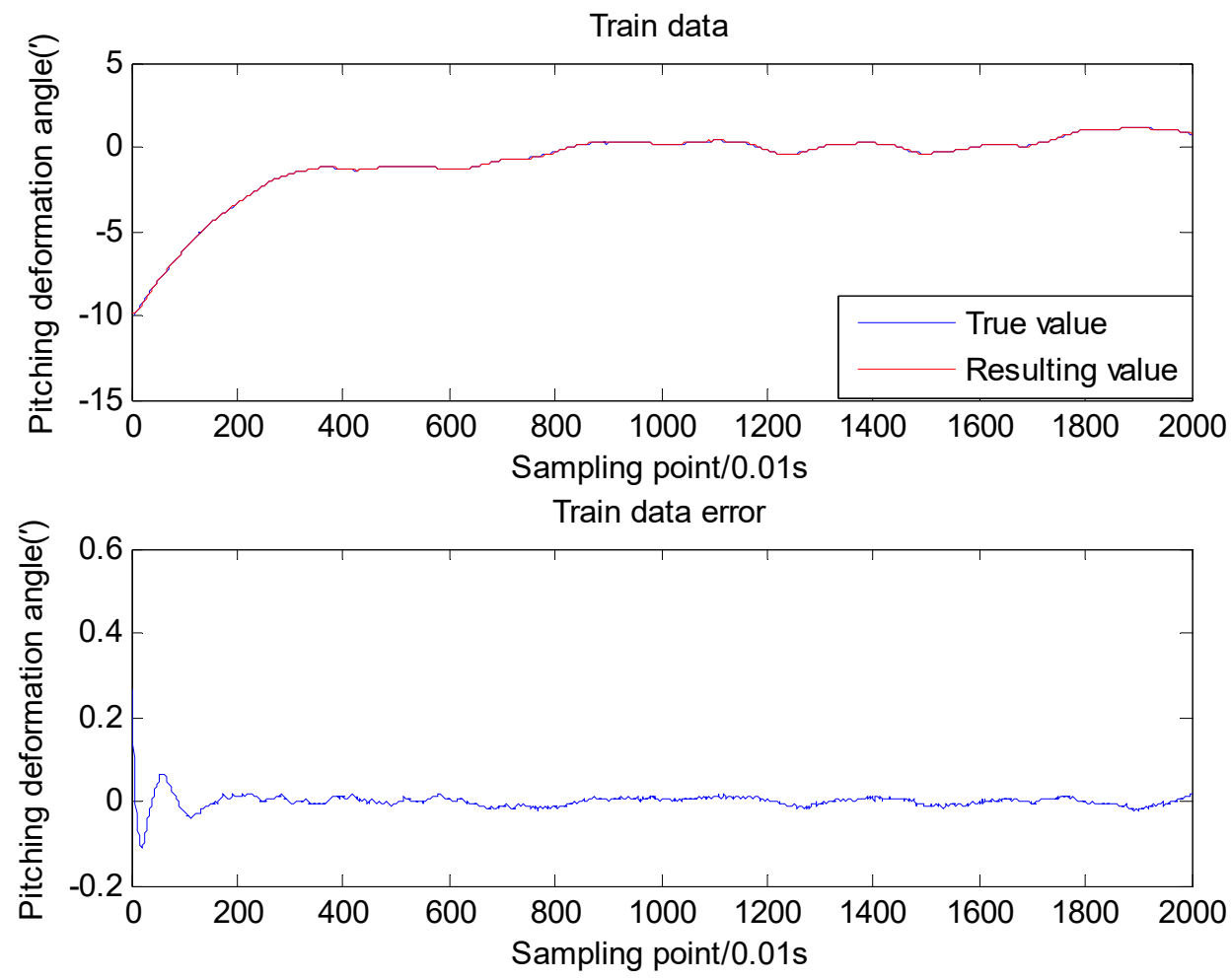

Fig.4 Training data and its error
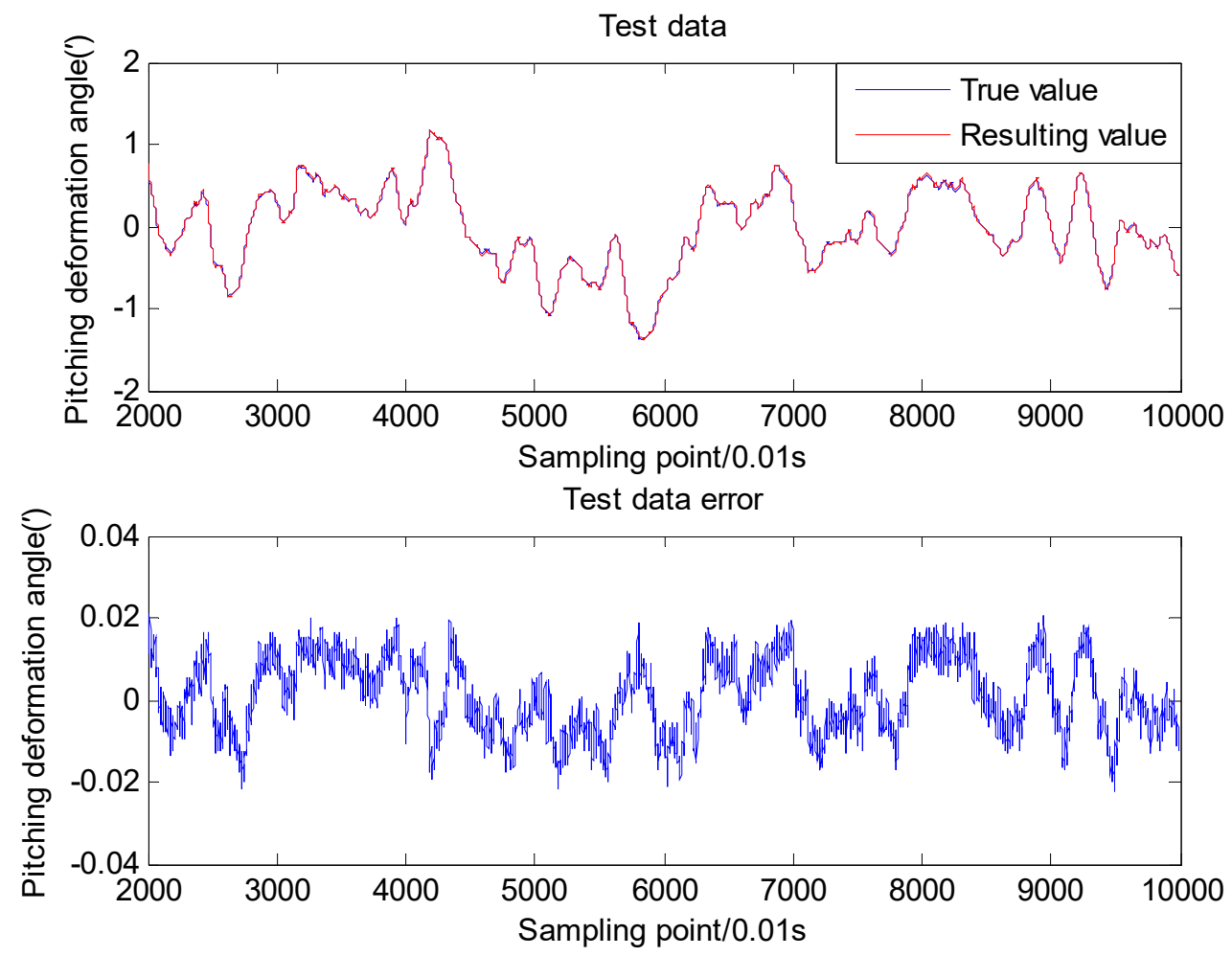

Fig.5 Test data and its error

As is shown in Fig.5, the deformation time series prediction method of large-sized flexible structure based on RBF neural network has a great prediction effect. This method can predict the dynamic deformation angle in a short period of time with high precision, with the error in the range of $0.02^{\prime}$. 


\section{Conclusion}

First of all, deformation mathematical model of large-sized structure based on mechanism is analyzed in this paper. Second, the deformation trend time series model is studied. Then the Radial-Basis Function (RBF) neural network is used to complete modeling and training process, realizing short-term deformation forecasting of large-sized structure. The simulation results show that the prediction method has a great prediction effect, which can predict the dynamic deformation angle in a short period of time with high precision, with the error in the range of $0.02^{\prime}$.

\section{References}

[1] Zhou Y X. One Technology of the Aircraft Wing Deformation Measurement in Flight-Test[J]. Measurement \& Control Technology, 2013.

[2] Zheng R C, Chen C Y, Yang G L, et al. Deck deformation measurement of large-sized ship based on LSE[J]. Journal of Harbin Institute of Technology, 2009, 41(9):141-144.

[3] Liu H B, Sun C, Zhang Y Q, et al. Hull deformation measurement for spacecraft TT\&C ship by Photogrammetry[J]. Science China Technological Sciences, 2015, 58(8):1339-1347.

[4] Wei W U, Qin S Q, Yang Y T, et al. Error suppression mechanism for ring laser gyro in ship deformation measurement[J]. Zhongguo Guanxing Jishu Xuebao/journal of Chinese Inertial Technology, 2014, 22(5):665-670.

[5] Zheng J X, Qin S Q, Wang X S, et al. Attitude matching method for ship deformation measurement[J]. Zhongguo Guanxing Jishu Xuebao/journal of Chinese Inertial Technology, 2010, 18(2):175-180.

[6] Majeed S, Fang J. Comparison of INS based angular rate matching methods for measuring dynamic deformation[C]// International Conference on Electronic Measurement \& Instruments. IEEE, 2009:1-332-1-336.

[7] Yan X B, Wang Z, Yu S H, et al. Time series forecasting with RBF neural network[C]// International Conference on Machine Learning and Cybernetics. 2005:4680-4683 Vol. 8.

[8] Ying-Sheng H E, Duan M X. Time Series Prediction Based on RBF Neural Network[J]. Journal of Jishou University, 2008. 